\title{
4 Wahn aus Sicht der Neuen Phänomenologie
}

\author{
Andrea Moldzio
}

\subsection{Grundlagen der Neuen Phänomenologie}

Die Neue Phänomenologie wurde von dem Kieler Philosophen Hermann Schmitz (1980) begründet. Das Anliegen der Neuen Phänomenologie ist es, die spekulativen Konstruktionen der traditionellen Philosophie zu verlassen und die Philosophie wieder auf der Ebene der ursprünglichen und jedem zugänglichen Lebenserfahrung des Menschen zu verankern. Getreu der phänomenologischen Maxime „zu den Sachen selbst“ ermöglicht ein neues und an der konkreten Lebenserfahrung geeichtes Begriffssystem, alltägliche Phänomene wie leibliche Regungen, Atmosphären, Gefühle etc. besser zu verstehen. Eine so „geerdete“ Abstraktionsbasis erhöht auch die wissenschaftliche Anschlussfähigkeit für andere wissenschaftliche Disziplinen, wie insbesondere die Psychiatrie. Sie bietet fruchtbare Anregungen und Denkanstöße, um die Komplexität anthropologischer Phänomene besser zu verstehen (vgl. auch Moldzio 2002, 2004a, b). 3

Die Neue Philosophie umfasst vier anthropologische Grundsäulen, nämlich

- Subjektivität,

- Leiblichkeit,

- Gefühl und

- Situation,

die hier nur kurz, soweit sie für die Falldarstellung notwendig sind, beschrieben werden (vgl. auch Moldzio 2004a).

3 Dieser Beitrag basiert auf einem bereits veröffentlichten Text der Autorin (Moldzio 2004a). Mit freundlicher Genehmigung des Verlages Königshausen und Neumann. 
Die erste Säule, die Subjektivität, beinhaltet die revolutionäre These, dass das Subjektive primär nicht in Form von Subjekten, sondern in Form von subjektiven Tatsachen vorhanden ist. Die Subjektivität wird dadurch der traditionellen Zugehörigkeit zu Subjekten entzogen und stattdessen den Tatsachen zugeordnet.

Von subjektiven Tatsachen kann gesprochen werden, wenn höchstens einer sie im eigenen Namen aussagen kann, wie Schmitz oftmals an dem Beispiel „Ich bin traurig!" exemplifiziert hat (Schmitz 1997, S. 15ff.; 1995, 1994, S. 59; 1992). Subjektive Sachverhalte sind an das affektive Betroffensein gebunden, d.h., sie müssen jemandem nahegehen und ihn in eindeutiger Ichbezogenheit betroffen machen oder ergreifen. Wenn ich ,ich“ sage und damit ausdrücke, dass es sich in unverwechselbarer und unvertretbarer Weise um mich persönlich handelt, verdeutlicht sich darin die Subjektivität von subjektiven Tatsachen. Diese Form der Subjektivität ist das Reich des affektiven Betroffenseins (Moldzio 2004a).

Sie stehen im Gegensatz zu den objektiven Tatsachen, die jeder, der genügend Kenntnisse darüber besitzt, aussagen kann.

Die zweite Säule der neophänomenologischen Anthropologie bildet der Leib des Menschen. Der cartesianische Dualismus mit seiner Trennung von Psyche (res cogitans) und Soma (res extensa) prägt bis heute unser Menschenbild, wie es auch in dem Begriff „Psychosomatik“ zum Ausdruck kommt. Dabei geriet der Leib als ein diffuses, nicht zuordenbares Etwas in Vergessenheit. Schmitz versteht unter „leiblich“ zum einen das Spüren am eigenen Körper ohne Zuhilfenahme der fünf Sinne oder des perzeptiven Körperschemas ${ }^{4}$, und zum anderen die leibliche Kommunikation, die über das Spüren des eigenen Leibes weit hinausreicht, um mit der Mitwelt in Kontakt zu treten. Jedoch wird der Leib nach Schmitz (1998b) nicht als etwas Statisches oder rein Privatives verstanden, sondern als etwas sehr Dynamisches, das mittels leiblicher Kommunikation über das Subjekt hinausgreift.

Schmitz betont, ,daß Leiblichkeit als Struktur nicht auf einzelne menschliche und tierische Leiber verteilt ist, sondern diese von Anfang an übergreift und soweit reicht wie der Horizont derWahrnehmung." (Schmitz et al. 2002, S. 204).

Die leibliche Dynamik spannt einen Bogen zwischen den beiden Endpolen der Weite und Enge. Die Enge des Leibes wird z.B. bei Angst oder Schreck deutlich, wo der Mensch ganz in sich zusammenfährt (vgl. Schmitz 1998a, S. 173ff.). Im Schreck wird der Leib unabhängig der anatomischen Körpergrenzen vielfach als klein und kompakt gespürt. 5

Die Angst ist eine in Psychiatrie und Psychosomatik häufig geschilderte leibliche Regung, die sich oft um Leibesinseln wie die Magengegend (,mein Magen krampft sich vor Angst zusammen“), Halsgegend („es schnürt mir die Kehle zu“) oder linke Thoraxhälfte („Druck- und Engegefühl in der Brustgegend“) zentriert. ${ }^{6}$

Der leiblichen Enge steht die leibliche Weite entgegen, wie sie sich z.B. beim Versinken in den Schlaf, bei der Wollust oder in Trance äußert. In der Weite dezentriert sich der Mensch durch die zentrifugalen Kräfte der Weitung. Wird der Mensch am Enge-

4 Dem perzeptiven Körperschema liegt ein physiologistisch reduziertes Modell des Körpers zugrunde, der durch Lage- und Abstandsbestimmungen sinnlich erfassbar ist. Dieses Modell ist in der Medizin dominierend.

5 Als Beispiele gespürter Enge führt Schmitz (1985) folgendes auf: „Die Engung überwiegt beim Schreck, Angst, Schmerz, gespannter Aufmerksamkeit, Beklommenheit, Hunger, dumpfem Zumutesein ..." (S. 82)

6 Interessanterweise leitet sich das Wort Angst auch etymologisch vom indogermanischen Wort ang(h)os = Enge, Bedrängnis ab. 
pol auf die Spitze seiner fünffach entfalteten Gegenwart in ein Hier, Jetzt, Dieses, Dasein und Ich geworfen, wird er am entgegengesetzten Weitepol, außerhalb seines Zentrums, von sich weg getrieben. So kann er im Extremfall, wie es zum Beispiel in tiefen Trancezuständen der Fall sein soll, zu keinerlei Selbstzuschreibungen im Rahmen einer Ich-Identität mehr fähig sein, da er nur noch Weite und Einssein mit dem Kosmos verspürt.

Schreck oder Angst sind Beispiele elementar-leiblichen Betroffenseins in primitiver Gegenwart, die den Menschen auf sich selbst zurückwerfen und an die Enge seiner leiblichen Gegenwart fesseln. Die primitive Gegenwart des Menschen, die er auch mit den Tieren gemeinsam hat, verweist ihn auf seine naturgegebene Basis.

„Das Zusammenschreckenkönnen, das Plötzliche, das Abreißen von Kontinuität in primitiver Gegenwart scheidet das Tier von der Pflanze, die Entfaltung der Gegenwart den Menschen (...) vom Tier." (Schmitz 1985, S. 100; 1997, S. 50).

Eine besonnene Differenzierung bezüglich Ort, Zeit und Charakter der angst- oder schreckauslösenden Ursache ist im Daseinsmodus der primitiven Gegenwart nicht möglich.

Die Erhebung des personalen Subjekts aus der primitiven Gegenwart bezeichnet Schmitz (1990a, b) als personale Emanzipation, seinen Rückfall an die primitive Gegenwart als personale Regression.

Sowohl die in die primitive Gegenwart führende personale Regression als auch die in die entfaltete Gegenwart führende personale Emanzipation sind für die Entwicklung der Persönlichkeit des Menschen gleichermaßen wichtig. Einem Leben ohne personale Regression, d.h. ohne affektive Betroffenheit und eigenleibliches Spüren, fehlt der Biss, die gelebte Fülle des Lebens mitsamt seinen Höhen und Tiefen. Eine hölzerne Verstiegenheit ist die Folge. Einem Leben ohne personale Emanzipation fehlen reife Besonnenheit, Idealbildung und Kritikfähigkeit (vgl. Schmitz 1990a, S. 105f.; 1990b, S. 156f.). Die Reifung der Persönlichkeit vollzieht sich in Form eines dynamischen Wechselspiels als „instabile Mannigfaltigkeit“ (Schmitz) zwischen primitiver und entfalteter Gegenwart, und kann dabei viele Niveaus und Stile annehmen. Da primitive und entfaltete Gegenwart miteinander verknüpft sind, können Störungen in der Leiblichkeit mit Störungen in der Persönlichkeitsentwicklung des Menschen zusammenhängen.

Der Leib ist alles andere als ein statisches Abstraktum, welches über die Grenzen des Subjekts nicht hinausreicht, er kann als „universaler Resonanzboden“ (Schmitz 199ob, S. 116), der die Kommunikation mit Anderen ermöglicht, verstanden werden.

Die dritte Säule für das menschliche Selbstverständnis stellen die Gefühle dar. Seit Platon und Aristoteles werden Gefühle als Seelenzustände betrachtet, die einer abgegrenzten Sphäre des Subjekts angehören, welche im allgemeinen als „Seele“, „Psyche“ oder „Bewusstsein“ bezeichnet wird. Der sogenannten Seele als Ursprungsort der Subjektivität werden verschiedene subjektive Regungen wie Gefühle, Triebe oder leibliche Regungen zugeordnet.

Im Gegensatz dazu kommt Schmitz zu einer völlig anderen Theorie der Emotionen, nach welcher Gefühle keine Seelenzustände, sondern „räumlich ergossene Atmosphären“ sind, die über die Subjektgrenzen hinausgreifen und auch leiblich gespürt werden können. 
Schmitz (1997, S. 153) stellt folgende drei Thesen über Gefühle auf:

1. Gefühle sind leiblich ergreifende Mächte.

2. Gefühle sind räumlich, aber ortlos ergossene Atmosphären.

3. Gefühle haben Autorität.

Eine feierliche Sonntagsatmosphäre oder eine bedrückende bzw. gespannte Atmosphäre kann den gesamten Raum samt anwesenden Personen umspannen und ist unmittelbar leiblich spürbar. So kann sich z.B. das Gefühl der Freude als motorisches Emporspringen, Hüpfen oder „beflügeltes“ Gehen zeigen, indem mit spielender Leichtigkeit der Widerstand der Schwerkraft wie außer Kraft gesetzt erscheint. Der fröhliche Mensch ist in seinem leiblichen Befinden in eine seine Subjektgrenzen übersteigende Atmosphäre der Leichtigkeit und Freude eingebettet, in der ihm die Überwindung von Widerständen mühelos gelingt. Demgegenüber erscheint dem traurigen Menschen die gesamte Umgebung mit eigentümlicher Schwere durchzogen, sodass bei schweren depressiven Zuständen allein das Verlassen des Bettes einen unüberwindlichen Kraftaufwand darstellen kann. Atmosphären entziehen sich physikalischer Messbarkeit. Dass Gefühle über die privative Innenwelt hinausgehen, wird auch an Gefühlskontrasten deutlich: Wenn ein Fröhlicher unvermittelt in eine Cruppe trauriger Menschen hineingerät, schlägt ihm oft eine Atmosphäre niederdrückender Schwere und Trauer entgegen. Depressive Menschen strahlen oft schon von weitem eine Atmosphäre der Schwermut aus, die im persönlichen Kontakt bei entsprechender Sensibilität des Gegenübers auch leiblich gespürt werden kann und beispielsweise derb-lustige Scherze geradezu imperativisch verbietet. Dies verdeutlicht die Autorität von Gefühlen.

Bei einem Zusammentreffen von unverträglichen Gefühlen wird deutlich, dass Gefühle ganzheitliche Atmosphären sind, die nicht nur die jeweilige Person, sondern die gesamte Situation der beteiligten Personen ergreifen. Insofern sind Gefühle ortlose, sich uferlos in die Weite erstreckende Atmosphären. Erst das Fühlen von Gefühlen (im Unterschied zum bloßen Wahrnehmen), verbunden mit leiblichen Regungen, macht affektives Betroffensein möglich, bei dem der Mensch durch sein Gefühl auch ergriffen wird. Gefühle ergreifen den Menschen durch leibliche Regungen und manifestieren sich beim Fühlenden als leiblich spürbares Betroffensein. Niedergeschlagenheit oder Kummer drücken sich mit erstaunlicher Gebärdensicherheit im gesamten Ausdrucksverhalten des Menschen aus, ohne dass dies erst erlernt werden muss.

Die vierte anthropologische Säule wird durch den Begriff der Situation gebildet, der ein Schlüsselbegriff der Neuen Phänomenologie ist. Die klassische Ontologie geht von einer Subjekt-Objekt-Dichotomie aus, nach der Subjekt und Objekt verschiedene Substanzen sind, die „irgendwie“ miteinander in Beziehung treten können. Die Frage, wie denn nun das Objekt zum Subjekt komme oder umgekehrt, ist daher stetiger Streitpunkt der Erkenntnistheorie. Schmitz versucht, die starre Subjekt-Objekt-Spaltung und die Trennung von Innen- und Außenwelt durch den Situationsbegriff aufzulösen.

Zur Säule der Situation gehört auch die persönliche Situation, unter der Schmitz die Persönlichkeit des Menschen samt seiner Lebensgeschichte versteht. Die persönliche Situation formiert sich aus dem Charakter, zu dem Erinnerungen, Standpunkte, Gesinnung, Leit-, Wunsch- und Schreckbilder und Lebenstechniken gehören; aber auch sein Temperament, das sich als persönliche leibliche Disposition und Schwingungsfähigkeit manifestiert (Schmitz 199ob, S. 169; 1994, S. 178ff.). 
„Die persönliche Situation ist das, was man gewöhnlich im vagen Sinn die Persönlichkeit eines Menschen nennt, wobei man diese als Gefüge von Eigenschaften missversteht, während es sich vielmehr um ein dynamisches Ganzes chaotischer Mannigfaltigkeit handelt, aus der Spitzen der Individualität (...) herausragen." (Schmitz 1990b, S. 168).

Die persönliche Situation entwickelt sich im Spielraum zwischen der personalen Regression und Emanzipation, sodass sich eine Lebensgeschichte bilden kann. Besonders Situationen tiefer affektiver Betroffenheit, wie sie beispielsweise bei psychischen Traumen vorkommen, die das Niveau personaler Emanzipation erschüttern, können die Lebensgeschichte fortan prägen.

Neben retrospektiven und präsentischen Inhalten hat die persönliche Situation auch einen prospektiven Gehalt (Schmitz 1997, S. 57; 199ob, S. 169f.). Letzterer kann als Entwurf zukünftiger Lebensmöglichkeiten, besonders bei schwierigen Lebensentscheidungen, relevant werden. Manchen Krisensituationen liegen mangelnde prospektive Lebenskonzepte zugrunde, die im Nachdenken und im gemeinsamen Gespräch erst gefunden bzw. entwickelt werden müssen, um die aktuelle Situation zu meistern.

\subsection{Definition des Wahns aus Sicht der Neuen Phänomenologie}

Nach diesen Hintergrundinformationen können wir uns nun dem Wahn widmen. Nach dem auf der Neuen Phänomenologie basierenden Verständnis der Autorin könnte der Wahn folgendermaßen definiert werden.

Wahn ist die Wahrnehmung vielsagender (Leit-)Eindrücke auf dem Boden der jeweiligen persönlichen Situation, die auf einem bestimmten Niveau personaler Emanzipation eingerastet ist, den Status einer subjektiven Tatsache hat (die jedoch für eine objektive Tatsache gehalten wird) und in der Regel von der soziokulturellen Bezugsgruppe nicht geteilt wird.

In dieser Definition sind folgende Implikationen enthalten:

1. Die Störung des Wahns liegt auf der Ebene der Wahrnehmung und nicht, wie traditionell von Griesinger und Kraepelin definiert, auf der Ebene des Denkens oder Urteilens. Aber nicht die Wahrnehmung als solche ist gestört, sondern die (Un-)Beweglichkeit ihrer weiteren Verarbeitung. Die Wahrnehmung beschränkt sich nicht auf die sinnliche Wahrnehmung äußerer Gegenstände, sondern umfasst auch ein sensibles Wahrnehmen eigener Zuständlichkeiten und Anmutungen, das Erfassen der eigenen persönlichen Situation und seiner eigenen momentanen Stellung zum Anderen und zur Welt. Die Wahrnehmung kann sich sowohl auf die eigene persönliche Situation wie auch auf die Welt beziehen und ist dann in ihrer Veränderung kompatibel mit Janzariks Ausdruck der „Entzügelung des impressiven Wahrnehmungsmodus“. Daher kann der Wahninhalt zum einen auf die Umgebung bezogen werden, dass zum Beispiel die Welt untergehen wird, und zum anderen auf die eigene Person, dass der Patient sich beispielsweise für eine andere Person hält.

2. Es handelt sich beim Wahn um die Wahrnehmung vielsagender (Leit-)Eindrücke. Das Vielsagende imponiert besonders in der Wahnstimmung. In dieser verdichtet sich affektiv die chaotische Mannifaltigkeit des vielsagenden Eindrucks zur beängstigenden Atmosphäre. Auch lässt sich, wie später gezeigt wird, am 
Beispiel der Wahnwahrnehmung verdeutlichen, wie Eindrücke plötzlich vielsagend werden und statt einer sachlich-abgegrenzten Bedeutung einen vielsagenden und chaotischen Bedeutungshorizont entwickeln. So nahm ein Patient einen durch die Tür schreitenden Mann nicht als eben diesen wahr, sondern als einen Drahtzieher in einem gefährlichen Spionageakt, der durch sein Hindurchgehen durch die Tür den Anderen bedeuten will, dass es losgehen kann. Die Einfachheit der Handlung (ein Mann geht durch eine Tür) wird durch ein komplexes, oft systematisiertes Gefüge von vielsagenden Eindrücken ersetzt.

3. Die Wahnentstehung auf dem Boden der persönlichen Situation umfasst, dass der Wahn aus den jeweiligen retrospektiven, präsentischen oder prospektiven Anteilen gespeist wird, wie z.B. aus den konkreten Ängsten, Befürchtungen, Wünschen, Plänen vor dem Hintergrund der gesamten Persönlichkeit in ihrer Einbettung in gemeinsame Situationen. Der Wahn schöpft seine Themen aus subjektiven Sachverhalten, Programmen und Problemen und umfasst daher alle Gebiete, die dem Menschen persönlich nahegehen, wie Tod, Gott, Angst, Liebe, Freundschaft, das Gute und Böse, Freiheit, Abhängigkeit etc. Vor diesem Hintergrund der persönlichen Situation entstehen dann Beeinflussungsoder Verfolgungswahn, Schuld-, Liebes- oder Größenwahn. Dabei nimmt der Wahn nicht Maß an der Realität, sondern an der eigenen persönlichen Situation, an die er fixiert ist.

4. Die Wahrnehmung ist auf einem bestimmten Niveau personaler Emanzipation mit hohem Evidenzcharakter „eingerastet“.

Dies ist das eigentlich charakteristische Merkmal des Wahns. Eine bestimmte Wahrnehmung oder ein Urteil erhalten den Anstrich der Letztgültigkeit, der unhinterfragbaren Entschiedenheit und unbeugsamen Absolutheit. Durch dieses „Einrasten“ resultiert die für den Wahnkranken typische Unkorrigierbarkeit und Unbeeinflussbarkeit. Wer auf einer bestimmten Position fixiert ist, ist unfähig zum Überstieg (Conrad 1971) und kann auch keine Kontingenz zulassen. Die fixierte Gewissheit schließt jeden Zufall aus.

Der Evidenzcharakter subjektiver Tatsachen bewirkt die absolute Gewissheit des Bescheidwissens, des selbstverständlich Offensichtlichen und Wahren, das intersubjektiv höchstens geteilt, keineswegs jedoch diskutiert oder bezweifelt werden kann.

Das „Einrasten“ auf einem bestimmten Niveau personaler Emanzipation bedingt die Wahl des Wahnthemas, die Art und das Ausmaß der Wahnarbeit etc. Je höher das Niveau der personalen Emanzipation, desto wahrscheinlicher wird die Wahl eines quasi-rationalen Wahnthemas. So steht beispielsweise der Beeinflussungs- oder Beeinträchtigungswahn auf einem höheren Niveau personaler Emanzipation als der Liebes- oder Schuldwahn, die näher am Pol der personalen Regression liegen, da bei letzteren die Komponente der affektiven Betroffenheit wahnkonstitutiv wirkt. Gefühle der Liebe oder Schuld sind basale Gefühle des Menschseins überhaupt und tief im affektiven Betroffensein verwurzelt.

Gleichzeitig entspricht auch die Höhe des Niveaus personaler Emanzipation dem Ausmaß der Wahnarbeit. Je höher das personale Niveau, desto wahrscheinlicher ist es, dass viel Wahnarbeit in systematisierter Weise geleistet wird. Ein komplexes Wahnthema, welches in differenzierter Weise durchsystematisiert wird, spricht für ein hohes Niveau personaler Emanzipation. 
5. Der Wahn erhält den Status einer subjektiven Tatsache, d.h. einer Tatsache, die nur einer in seinem Namen aussagen kann. Sämtliche Bekehrungs-, Widerlegungs- und Überzeugungsversuche von Angehörigen oder Professionellen, dem schizophrenen Menschen den Wahn auszureden und von dessen Unrichtigkeit zu überzeugen, scheitern an dieser Diskrepanz zwischen objektiver und subjektiver Tatsache. Eine Argumentation gegen den Wahn verkennt den Charakter des Wahns als verabsolutierte subjektive Tatsache und erreicht lediglich die Ebene der objektiven Tatsachen, die jeder vertreten kann, wenn er entsprechend genug darüber weiß. Die Korrektur einer subjektiven Tatsache durch eine objektive ist phänomenologisch nicht möglich, da sich die phänomenalen Ebenen verfehlen. Auch ist der Patient wahnhaft in seiner präpersonalen Subjektivität fixiert, die nicht so einfach durch Einflüsse der personalen Subjektivität umgeformt werden kann. Das Oszillieren zwischen personaler und präpersonaler Subjektivität gelingt nicht mehr mühelos, sondern versteigt sich auf einer bestimmten Ebene, die - wahnhaft fixiert - nicht mehr verlassen werden kann. Dadurch wird das Betroffenmachende auf dem Boden präpersonaler Subjektivität direkt, nah und ganz konkret erlitten, ohne in distanzierende Abstandnahme auf dem Niveau personaler Emanzipation bearbeitet werden zu können. Das sich Andrängende und Betroffenmachende entäußert sich daraufhin in der Wahnbildung, wodurch das Bedeutsame implizit ausgestaltet wird.

Diese phänomenologische Wahndefinition im Blick können im Folgenden zwei spezielle Wahnphänomene betrachtet werden. Von der traditionellen Wahnforschung werden als hauptsächliche Wahnformen die Wahnwahrnehmung und der Wahneinfall voneinander unterschieden (Huber u. Gross 1977, S. 12).

\subsection{Wahnwahrnehmung}

Seit Jaspers steht das Phänomen der Wahnwahrnehmung im Mittelpunkt des Wahnproblems.7 An diesem Phänomen entzünden sich brennende Fragen über Wahrnehmung überhaupt, Verständlichkeit vs. Unverständlichkeit von Wahn, formale Strukturmerkmale oder das Wechselverhältnis von Subjekt und seiner Welt.

Der Begriff der Wahnwahrnehmung wurde von Jaspers (1913) bei der Thematisierung „primärer Wahnerlebnisse“, zu denen er Wahnwahrnehmung, Wahnvorstellung und Wahnbewusstsein zählt, eingeführt (S. 82ff.). Für ihn gehen Wahnwahrnehmungen vom „Erlebnis unklarer Bedeutungen bis zu klarem Beobachtungs- und Beziehungswahn“" (Jaspers 1913, S. 83).

Schneider präzisierte den Begriff und bezeichnete die Wahnwahrnehmung als Symptom 1. Ranges und insofern als besonders charakteristisch für die Schizophrenie. Nach Schneider redet man dann von

„Wahnwahrnehmung, wenn einer wirklichen Wahrnehmung ohne verstandesmäßig (rational) oder gefühlsmäßig (emotional) verständlichen Anlass eine abnorme Bedeutung beigelegt wird“ (Schneider 1949, S. 26; vgl. auch Huber u. Gross 1992).

7 Beispiele für Wahnwahrnehmungen: Eine Patientin der Autorin trug ständig einen Stein bei sich, der für sie Einstein - der Physiker - war und bedeutete, dass sie die Welt und all ihre Probleme errechnen muss. Ein anderer Patient entnahm einem Baugerüst die Bedeutung, dass die ganze Stadt abgerissen und umgebaut werden soll. 
Er fasste die Wahnwahrnehmung als formal zweigliedrig auf:

- Das 1. Glied geht vom Wahrnehmenden zum Gegenstand in allgemeiner Bedeutung und

- das 2. von letzterem zur abnormen Deutung; das 2. Glied ist unableitbar und unverstehbar (Schneider 1949, S. 28).

Beispielsweise sieht ein Patient ein Kreuz (1. Glied) und schlussfolgert aus dieser (richtigen) Wahrnehmung im zweiten Schritt (2. Glied), dass dies bedeutet, dass er umgebracht werden soll.

Schneider hält die Wahrnehmung als solche für ungestört, auf die sich anschließend eine abnorme Bedeutung aufsetzt. Für die Diagnostik der Wahnwahrnehmung sei nicht der Inhalt, sondern allein die zweigliedrige Struktur relevant.

Schmitz hält die Zweigliedrigkeit der Wahnwahrnehmung für unzutreffend, weil zum einen demnach auch Kinder Wahnwahrnehmungen hätten und zum anderen, weil es sich bei dem Schneiderschen Wahrnehmungsbegriff um eine sensualistische Reduktion der Wahrnehmung handelt. DieTrennung zwischen Wahrnehmung und Denken, wie es Schneider nahelegt, ist Ausdruck eines empiristischen Wahrnehmungsverständnisses. Dieses basiert auf dem physiologistisch-rationalistischen Irrtum, die Wahrnehmung auf einen sinnlichen Unterbau zu reduzieren, auf den anschließend ein Überbau von Bedeutungen aufgesetzt wird. Nach Schmitz besteht die genuine Form der Wahrnehmung, wie anfangs beschrieben, aus vielsagenden Eindrücken, die in der Regel aus ganzheitlichen Situationen bestehen. Eine zweigliedrige Wahnwahrnehmung ist phänomenologisch nicht nachweisbar (Schmitz 1990a, S. 465).

Blankenburg (1965) sah in der Wahnwahrnehmung eine „Entharmlosung“ der Welt, in der das Unauffällige auffällig wird. Der Patient kann sich nicht darauf einstellen, sondern ist im Sinne eines „Ein für Allemal“ darauf eingestellt. Dieses Eingestelltsein ist die eigentliche „Ver-rückung“(i.S. von „Einrasten“) des Schizophrenen. Dieser ist unfähig, empfangene Eindrücke integrativ zu verarbeiten, d.h. zu assimilieren. Die Beeindruckbarkeit und die Assimilationsfähigkeit stehen beim Gesunden im ausgewogenen Verhältnis, beim Schizophrenen bricht es auseinander. Für Blankenburg resultiert also die Wahnwahrnehmung aus einer gestörten Integrationsfähigkeit von empfangenen Eindrücken und wird durch die reduzierte Kommunikationsfähigkeit untermauert. Blankenburgs Position korreliert mit der Auffassung von Schmitz, dass durch die Lähmung der Persönlichkeit die Verarbeitung von Eindrücken und nicht deren Empfänglichkeit gestört ist.

\subsection{Wahneinfall}

Der Wahneinfall ist nach Schneider (1992) von der Wahnwahrnehmung vollkommen verschieden:

„Unter einem Wahneinfall verstehen wir Einfälle, wie den der religiösen Aufgabe, der besonderen Fähigkeit, der Verfolgung, des Geliebtwerdens. (...) Jeder Psychotische kann solche Wahneinfälle haben, und die Abgrenzung zu den Einfällen Nichtpsychotischer, zu ,überwertigen und Zwangsgedanken, ist begrifflich unmöglich. (...) Er (der Wahneinfall, Anm. der Verf.) besteht nicht in abnormem, anlasslosem Bedeutungserleben einer Wahrnehmung, sondern ist ein rein gedankliches Meinen." (Schneider 1992, S. 53). 
Schneider hält den Wahneinfall entgegen der psychiatrischen Tradition (und im Gegensatz zur Wahnwahrnehmung) in seiner Struktur für eingliedrig, da alles primäre Wahnerleben ein Erleben von Bedeutungen sei (Schneider 1992, S. 53f.). ${ }^{8}$

Phänomenologisch gesehen resultiert die für den Wahn typische Unkorrigierbarkeit durch objektive Tatsachen aus dem Verlust des kritischen Abwägens durch die Lähmung der Persönlichkeit. Dieses kritische Abwägen wird normalerweise durch verschiedene Niveaus personaler Emanzipation und Regression erreicht, die ein Mitgehen mit affektiver Betroffenheit und kritischer Abstandnahme ermöglichen. Erst dadurch kann eine Nuancierung der Gewissheit stattfinden. Es fehlt das Bewegliche, das Fließende, in dem Einstellungsvermögen, wodurch Niveauänderungen verhindert werden.

Einem Patienten mit Eifersuchtswahn ist es z.B. unmöglich, seine feste Überzeugung, von seiner Frau betrogen zu werden, auf dem Niveau der personalen Emanzipation kritisch zu hinterfragen und aufzugeben. Auch unmögliche Wahnsysteme können nicht mehr auf dem Boden der personalen Emanzipation reflektiert werden. Auch hier liegt eine Fixierung auf dem Niveau der präpersonalen Subjektivität vor mit Dominanz subjektiver Tatsachen, die durch objektive Tatsachen nicht zu korrigieren sind. Dadurch wird der Wahneinfall zu einem „Zu-Fall“, einer erlittenen Evidenz mit der Unfähigkeit, den „eingefallenen“ Sachverhalten, Programmen und Problemen wieder ihre bislang unbedingte Subjektivität abzustreifen. Diese Unfähigkeit, zwischen verschiedenen Niveaus der Subjektivität und Objektivität oszillieren zu können, ist das eigentlich Pathologische an dem Wahneinfall. Dadurch unterscheidet er sich u.a. auch von „abwegigen“ Ideen.

\subsection{Interpretation der Fallbeispiele aus der Perspektive der Neuen Phänomenologie}

Aufgrund der höheren Authentizität in der Selbstschilderung der Patientin, die sich für Sartre hält (s. Anhang), und der damit verbundenen besseren Eignung für phänomenologische Betrachtungen, wird dieser im Folgenden vorrangig betrachtet. Der geschilderte Fall des Patienten 1 („Ein Mordversuch als wahnhafte parasuizidale Handlung“") kommt im Schlussteil des Beitrages kurz zur Sprache.

Der interessante Fall der psychotisch gewordenen Philosophiestudentin (Patientin 2) beschreibt aus der Retrospektive mit einer gewissen Wahndistanz ein sehr fluides und buntes Wahnsystem, das in bestechend elaborierter und geordneter Weise von ihr so beschrieben wird, als ob sie ihre eigene Wahnkatamnese hätte schreiben sollen. Kaum vorhandene formale Denkstörungen und das Bemühen der Patientin, stets sinnreiche Verbindungen ihrer Wahneinfälle mit ihrer persönlichen Situation zu knüpfen und dabei die „Als-ob-Realität“ klar darzustellen, machen es dem Leser leicht, sich in den Wahn hineinzuversetzen. Zudem sind die sehr emotional aufgeladenen Themen wie Verliebtsein, Trennung, Schmerz, Tod, Hochzeit, die die Patientin intensiv wahrnimmt und schildert, als Identifikationsobjekte geeignet, die allgemeinen Themen menschlichen Seins anzurühren.

8 Für Jaspers hingegen sind beide Phänomene zweigliedrig. 
Aus Sicht der Neuen Phänomenologie liegen hier also Themen von höchstem affektivem Betroffensein vor, die das formale Denken zumindest zum Zeitpunkt der reflektierten Selbstschilderung fast unbetroffen lassen. Diese gesteigerte affektive Betroffenheit ist auch der springende Punkt in dem bunten Wahnsystem, das seinen Ausgangspunkt in der Zuspitzung der persönlichen Situation, die durch den Abschluss des Philosophiestudiums und die gleichzeitigen Trennungsabsichten zu dem einen Mann und Verliebtheitsgefühlen zu dem anderen, geprägt ist. Hier liegt wohl die Spitze bzw. Mitte des derzeitigen affektiven Betroffenseins vor, was sich in der emotionalen Dichte der Selbstschilderung widerspiegelt. Das quälende Gefühl von depressiven Gedanken und die schweren Versagensängste, die sich auch durch gesteigerten Alkoholkonsum nicht dauerhaft lindern ließen, mündeten nach Abgabe der Diplomarbeit in eine „entsetzliche Leere“, die den Boden für die Wahnentwicklung bildete. Diese sehr auf sich selbst bezogenen Gefühle der Depression, Angst und inneren Leere drängen aufgrund ihrer expansiven Kräfte darauf, die Grenzen des Subjekts zu überschreiten und sich nach außen auszubreiten. In diesem Sinne legt sich der nächste konzentrische Kreis affektiver Betroffenheit auf ihre Familie, bei der der Großvater im Verdacht steht, an den Naziverbrechen beteiligt gewesen zu sein. Mit dessen Taten identifiziert sich die Patientin im weiteren Verlauf so primär und intensiv, als würde ihr gleichzeitig die Schuld als Täter und die Chance als Retter durch Tötung ihrer Eltern auferlegt. Die Vergangenheit ihrer Familie ist das Nächste, was noch zu ihr gehört, mit dem sie sich identifizieren kann, ohne allzu weit ausholen zu müssen. Statt depressiver Leere oder Angst machen sich jetzt bei der Patientin Schuld- und Schamgefühle breit, die zumindest gegenüber den vorherigen Gefühlen den „Vorteil“ haben, dass sie nicht so diffus sind, sondern ein konkretes Objekt zum Ziel haben, welches vom eigentlichen Subjekt entfernter ist. Das Gefühl, aufgrund ihrer Familiengeschichte eine „Mitschuld“ zu tragen, ist immerhin erträglicher als das vernichtende Gefühl von sich ausbreitender depressiver Leere, die weder Anfang noch Ende oder gar Ziel hat. Zudem bietet es Möglichkeiten konkreter Auseinandersetzung, beispielsweise mit der eigenen Familiengeschichte oder der Rettung der Opfer.

Der nächste konzentrische Kreis des affektiven Betroffenseins nimmt von der eigenen Person oder Familiengeschichte mehr Abstand und nimmt die Romanhelden Sartres zum Anlass wahnhafter Identifizierung, die sich mit entsprechenden Halluzinationen verbinden. Diese Identifizierung ist jedoch nicht losgelöst von der persönlichen Situation, sondern wird zu ihr in Beziehung gesetzt, da sie „fühlte, dass die Atmosphäre dieser Werke meiner eigenen Lebenssituation glich“. Hier lockern sich bereits die persönliche Situation und die eindeutige Selbstzuschreibung, dass ich es bin, der denkt oder handelt. Die subjektive Tatsache, dass ich es bin und kein anderer, verliert ihre Gewissheit und droht zur objektiven Tatsache zu werden in dem Sinne, dass sie auch ein beliebig anderer sein könne. In spielerischer Identifizierung sieht sich die Patientin nicht mehr als sie selbst, sondern als Romanhelden in wechselnder Ausgestaltung, denen sie vermutlich zutraut, ihre innere Zerrissenheit schnell zu beenden.

Der nächste konzentrische Kreis umfasst dann nicht nur Philosophen, zu denen sie sich während ihres Studiums besonders hingezogen fühlte und die durch ihre Persönlichkeit und ihr Leben eine so ideale Basis zur spielerischen Identifikation mit sich selbst bieten wie Sartre, sondern erweitert sich auf berühmte Persönlichkeiten überhaupt, die markante Leidensgeschichten aufzuweisen haben. Hier besteht der größte Abstand zur eigenen persönlichen Situation, indem inzwischen nur wenige 
Gemeinsamkeiten oder bestimmte Eigenschaften, die diesen zugeschrieben werden bzw. ersehnt werden, ausreichen, um sich mit den berühmten Persönlichkeiten zu identifizieren.

Der psychotische Lösungsversuch der Integration verschiedenster Anteile gipfelt in der Hochzeit von Opfern und Tätern und der Verschmelzung von Mann und Frau, Subjekt und Objekt. Dem protopathischen Zerfließen in andere Personen wird versucht, Einhalt zu gebieten, indem alles wieder in einer großen Synthese zusammengeführt und dadurch auch greifbarer wird. Dem chaotischen Durcheinander, das in die völlige Selbstverlorenheit und Orientierungslosigkeit führt, wird stattdessen ein verdichtendes „Alles ist eins“-Konglomerat gegenübergestellt.

Nachdem auch die Entwicklung von Zwängen das psychotische System nicht mehr aufrecht zu halten vermag, bricht über den Weg der personalen Emanzipation mittels Selbstdistanzierung durch Drehen von Filmen und durch kurzfristige Selbsterhöhung mittels Verleihung des Nobelpreises für die Liebe zur Philosophie das ganze Wahnsystem in sich zusammen und endet erneut in einer tiefen Depression.

\section{Analyse des Fallbeispiel 1 nach phänomenologischen Wahnkriterien}

Doch schauen wir uns die eingangs beschriebenen phänomenologischen Wahnkriterien genauer an:

Die Störung auf der Ebene der Wahrnehmung verdeutlicht sich bei der Patientin ganz besonders in dem sensiblen Wahrnehmen von menschlichem Leid. Als 1. nimmt sie ihre Umgebung sensitiv verändert wahr (Leichen im Keller) und leidet mit den ermordeten Juden oder anderen berühmten Personen, später nimmt sie auch sich selbst als jemand anderes wahr (Existenz als Sartre, Thomas Bernhard, Oskar Wener und Romy Schneider). Immer ist der gemeinsame Nenner das existenzielle Leid. Hier findet auf dem Boden der eigenen persönlichen Situation, in der sich die Patientin als leidend erlebt, ein Ausweitung ursprünglich persönlichen Leides auf andere statt, um ihr eigenes empfundenes Elend zum einen symbolisch zuzuspitzen (,,jammernde Leichen“) und es zum anderen größenhaft zu überhöhen (Sartre sein) und auf andere zu verteilen.

Als 2. beeindruckt in der Selbstschilderung der Patientin die Wahrnehmung vielsagender Eindrücke, die sich in der bedrohlichen Wahnstimmung widerspiegeln. Der Gang in den Keller der schreienden Leichenberge wird als Vorhölle beschrieben. Die megaloman und phantastisch ausgestalteten Mordphantasien gegenüber ihren Eltern, die Höllenqualen durch Mitleiden der Schmerzen der anderen, werden angeregt durch die entzügelte Wahrnehmung von einem Quäntchen realer sowohl historischer als auch gegenwärtiger Begebenheiten im Leben der Patientin.

Das 3. Kriterium der Wahnentstehung auf dem Boden der persönlichen Situation wird auch aus der Schilderung der Patientin deutlich, indem alle Wahneinfälle und Wahnwahrnehmungen quasi als Zitat ihrer persönlichen Situation begannen. Die präsentischen Anteile manifestieren sich durch die studiumsbedingte Beschäftigung mit Sartre, die in die Vorstellung mündet, erst dessen Romanhelden und später Sartre selbst zu sein.

Die prospektiven Anteile ihrer persönlichen Situation befassen sich mit der sehr realen, wenngleich auch unausgesprochenen Frage, wie es nach Abschluss des Philosophiestudiums denn weitergehen soll. Zumal dann ein neuer Lebensabschnitt beginnt, 
der zudem noch von einer großen existenziellen Unsicherheit bedroht ist, da der Abschluss eines Philosophiestudiums bekanntlich nur ein sehr schwacher Garant für eine berufliche Festanstellung ist. Aus diesen Zukunftssorgen heraus entwickelt sich der beruhigende Wahneinfall, dass sie den Nobelpreis für Philosophie erhalten werde, woraufhin sich dann vermutlich auch alle weiteren Zukunftssorgen erledigt hätten.

Insgesamt sind die verschiedenen Wahnideen durch das Band größenhafter Selbstüberschätzung miteinander verbunden, indem sie beispielsweise die Aufgabe hat, die „Geschichte Österreichs aufzuarbeiten“, an ihren Eltern einen „Jahrhundertmord“ zu vollziehen, oder an der Überzeugung festhält, „eine der prägendsten philosophischen Gestalten des 20. Jahrhunderts“ zu sein.

Das 4. „Letztgültigkeitskriterium“ wird bei der Patientin sehr deutlich, indem ihre Wahneinfälle und Wahnwahrnehmungen mit größtem Evidenzcharakter behauptet und geglaubt werden, ohne in flexibler Abwägung auch andere Möglichkeiten mit einzubeziehen. Sie hält typischerweise eine Begründung und Rechtfertigung ihres Systems für überflüssig. Aufgrund der dem Wahnsystem inhärenten hohen subjektiven Evidenz erscheint ihr eine systemexterne Hinterfragung unnötig und ist ihr auch unmöglich. Die Patientin muss und kann sich für ihre Überzeugungen nicht mehr stellungnehmend einsetzen. Schizophrene Menschen haben quasi ein „undurchdringliches Korsett mit fixierten Bedeutsamkeiten“ an. Die Funktion dieses Korsetts oder dieses festen Gehäuses liegt in der sicheren Orientierung, die über jeden Zweifel und jede Verunsicherung erhaben ist. Der Preis dafür ist der Verlust der inneren Beweglichkeit, sodass die Patientin wie in einem goldenen Käfig von Bedeutungen gefangen ist. Sie verharrt in ihrem System „festgestellter“ Bedeutsamkeiten und argumentiert in ihrem Wahnsystem systemimmanent, da ihr der Überstieg, der Wechsel des Bezugsystems (Conrad 1971) nicht mehr gelingt. Die starre Eigenbeziehung der Patientin macht sie so unkorrigierbar und wahngewiss.

Gleichzeitig ist sie hier in der Wahl ihrer Wahnthemen sehr nah an dem Pol personaler Regression, da die beiden dominanten Gefühle der Liebe und Schuld sehr archaische und basale menschliche Themen sind, die in diesem Fall sehr systematisiert und intellektualisierend verarbeitet werden.

Das damit eng verbundene 5. Kriterium der Verabsolutierung subjektiver Tatsachen beschreibt phänomenologisch die bereits von Jaspers beschriebene hohe subjektive Gewissheit, die von den anderen als objektive Tatsache nicht geteilt wird. Diese wahninhärente Verabsolutierung subjektiver Tatsachen ist weder korrigierbar noch mit anderen teilbar und im wahrsten Sinne des Wortes eine „erlittene“ Evidenz.

Ganz anderes gestaltet sich das Wahnsystem von Patient 1 (s. Anhang), das sehr phantastisch, bizarr-manieriert anmutet und dabei wenig geordnet, durchsystematisiert und intellektuell durchdrungen ist. Sein Wahnsystem scheint wenig mit seiner realen persönlichen Situation zu tun zu haben und auch kaum affektiv aufgeladen $\mathrm{zu}$ sein. Man könnte fast meinen, dass die Wahnbiografie das Negativ der Realbiografie wäre. Während er im wirklichen Leben höchstens zu Prostituierten Kontakt hat, ist er in seiner Wahnbiografie unzählige Ehen mit „26 und mehr Kindern und 120 Enkelkindern auf der ganzen Welt verstreut“, eingegangen. Als reales Kind sehr einfachen Verhältnissen entsprungen, in denen er, wie sich vermuten lässt, eine eher 
unbedeutende Rolle gespielt hat, zentriert sich seine Wahnbiografie ausschließlich um größenhafte Identitäts- und Abstammungsthemen.

Aufgrund fehlender intellektueller Möglichkeiten, sich mit seiner persönlichen Situation so auseinanderzusetzen, wie der „Sartre-Patient“, bleibt ihm nur die Möglichkeit der Selbstinszenierung durch Leibhalluzinationen (Kugel im Herzen) als Symbolisierung seines Leidensdruckes oder zum Schluss die Selbsttötung via Getötetwerden. Hier ist der einzige Hinweis, dass der Patient leidet, zumindest unter dem schmerzhaften Projektil, und den Wunsch hat, von seinem Leid erlöst zu werden.

Worunter er jedoch genau leidet, wie seine persönliche Situation, seine Gefühle, sein Denken oder seine Leiblichkeit genau aussehen, geht aus der Fallschilderung nicht hervor. Auch gibt die reale Biografie des Patienten, die auffällig unauffällig ist, hierfür keinerlei Anhaltspunkte. Somit müssten für eine Analyse der Raum der phänomenologischen Beschreibungen verlassen und in den Bereich des Spekulativen ausgewichen werden. Dann würde man aber selbst in den Bereich wahnhaft-mystischer Spekulationen übergehen und sich jedenfalls nicht mehr auf dem Gebiet der Neuen Phänomenologie bewegen.

\section{Literatur}

Blankenburg W (1965) Zur Differentialphänomenolgie der Wahnwahrnehmung. Nervenarzt 36, 285-297

Conrad K (1971) Die beginnende Schizophrenie. Thieme Stuttgart

Huber G, Gross G (1977) Wahn. Eine deskriptive phänomenologische Untersuchung schizophrenen Wahns. Enke Stuttgart

Huber G, Gross G (1992) Die klinische Psychopathologie von Kurt Schneider. In: Janzarik W (Hrsg.) Psychopathologie als Grundlagenwissenschaft. Enke Stuttgart

laspers K (1913) Allgemeine Psychopathologie. 9. Unveränderte Auflage. Springer Berlin Heidelberg

Moldzio A (2002) Ein phänomenologischer Blick auf schizophrene Psychosen. In: Ertl M, Keintzel B, Wagne P (Hrsg.) Ich bin tausend Ich. Probleme, Zugänge und Konzepte zur Therapie von Psychosen. 236-244. Facultas Wien

Moldzio A (2004a) Schizophrenie - eine philosophische Erkrankung? Königshausen \& Neumann Würzburg

Moldzio A (2004b) Philosophie der Psychosen - vom Symptom zum Phänomen. In: Bock T, Dörner K, Naber D (Hrsg.) Anstöße. Zu einer anthropologischen Psychiatrie. 72-83. Psychiatrie Verlag Bonn

Schmitz H (1980) Neue Phänomenologie. Bouvier Bonn

Schmitz H (1985) Phänomenologie der Leiblichkeit. In: Petzold H (Hrsg.) Leiblichkeit, philosophische, gesellschaftliche und therapeutische Perspektiven. 71-106. Junfermann Paderborn

Schmitz H (1990a) System der Philosophie. Band IV: Die Person. Bouvier Bonn

Schmitz H (1990b) Der unerschöpfliche Gegenstand. Bouvier Bonn

Schmitz H (1992) Thesen zur phänomenologisch-philosophischen Fundierung der Psychotherapie. In: Kühn R, Petzold H (Hrsg.) Psychotherapie und Philosophie. 551-568. Junfermann Paderborn

Schmitz H (1994) Neue Grundlagen der Erkenntnistheorie. Bouvier Bonn

Schmitz H (1995) System der Philosophie. Band III, vierter Teil: Das Göttliche und der Raum. 2. Auflage. Bouvier Bonn

Schmitz H (1997) Höhlengänge. Akademie Verlag Berlin

Schmitz H (1998a) Der Leib, der Raum und die Gefühle. Edition Tertium Ostfildern

Schmitz H (1998b) System der Philosophie. 2. Band, 1. Teil. Der Leib. Bouvier Bonn

Schmitz H, Marx G, Molzio A (2002) Begriffene Erfahrung. Beiträge zur antireduktionistischen Phänomenologie. Ingo Koch Verlag Rostock

Schneider K (1949) Zum Begriff des Wahns. Fortschr Neurol Psychiatr 17, 26-31

Schneider K (1992) Klinische Psychopathologie. Thieme Stuttgart 